\title{
Impact of Protein Diet Supplements on Athletes
}

\author{
Athar $\mathbf{M}^{1 *}$ and Mohsin $\mathbf{M}^{2}$ \\ ${ }^{1}$ Assistant Professor, Department of Forensic Science, Institute for Excellence in Higher \\ Education, Bhopal, India \\ ${ }^{2}$ Undergraduate Student of Forensic Science, Department of Forensic Science, Institute for \\ Excellence in Higher Education, Bhopal, India
}

\section{Commentary}

Volume 6 Issue 2

Received Date: March 22, 2021

Published Date: April 05, 2021

DOI: $10.23880 /$ fsnt-16000264

*Corresponding author: Mohammad Athar, Assistant Professor, Department of Forensic

Science, Institute for Excellence in Higher Education, Bhopal, India, Email: athar.fsl48@gmail.com

\section{Abstract}

Protein supplements are one of the most commonly consumed nutritional supplements, whether by professional athletes or amateurs, even by those who use them for aesthetic purposes instead of sporting ones. The thirst for becoming better is increasing day by day and so is the quest for competition. Sports have been one of the greatest of platforms to showcase one's talent, thus leading to constant hustle for getting better day by day. To improve the performance, diet and physical appearance of the body i.e. muscle growth of athletes, sports nutrient products are developed. The use of these protein supplements in diet has increased the physiological requirement of protein but whether the use of these supplements is having positive or negative effect on the host is still a topic of debate for many. Large amount of protein derived constituents end up in the large intestine in case of high protein diets as compared to medium or low protein diets, leading to more bacterial amino acid metabolism in colon having positive as well as negative effects on the host. We, here, will try to summarize the impact of protein diet supplements on athletes.

Keywords: Protein \& Nutritional Supplements; Health Impacts; Sports Professionals

\section{Introduction}

Product consumed for the purpose of supplementing the diet with one or more ingredients is known as dietary supplement. Nutritional supplements commercially are classified into 3 types as weight loss agents, body building supplements and energy and performance boosters. For the purpose of body building and performance enhancements amino acid and protein supplements are widely used where as high protein and low carbohydrate supplements are used for weight loss purposes. High demand for dietary supplements in athletes has given birth to multibillion dollar industry; the market of dietary supplements in India is estimated to be around $\$ 3$ billion. The type and amount of dietary supplement used significantly varies from person to person specially the type of sports one is indulged in and on the metabolic state of an individual. Protein has always been an essential supplement of human diet due to its numerous advantages such as securing growth in infants, building body mass, improving physical performance, improving bone and muscle metabolism and maintaining normal nervous system. the use of protein supplement has increased to maintain required amount of protein synthesis and energy production, sufficient immune function in the multi stress condition or prolonged exercise. With the increase in time and intensity of athletic performance the quantity of protein in diet should also increase throughout the day including the time before and after workout so as to maintain the regular supply of 


\section{Food Science \& Nutrition Technology}

essential amino acids in the body, to maintain adequate amount of these amino acids in food many supplements are developed. For instance valine, leucine and isoleucine are some branched chain amino acids (BCAA) which are required by sports professionals to minimize muscle soreness to improve the performance during workout. On the other hand whey protein supplements are popular for sports professionals due to its fast absorption and digestion. Highest BCAA content of natural protein source is found in whey protein which stimulates muscle protein synthesis in a dose - dependent manner.

Protein supplements are quite popular in teenage groups for the purpose of muscle building and mass gain for better physical appearances. However the scientific evidence for these protein supplements to prove their pharmacological utility is very low but on the contrary signifies high chances of severe effects. Along with claiming high utility most of the supplement manufacturing companies do not highlight their severe effects, an increased risk of cardiovascular, hematological and dermatological side effects by the use of these supplements have been demonstrated by various studies. It has been found that around $10-25 \%$ of these supplements contain forbidden substances. Regular gym goers too consume protein, amino acid and creatine supplements along with other protein rich foods without any guidance from professional nutrition experts. Regular gym attendees' work load generally does not match with that of a professional athlete and so it is often recommended that the requirement of protein for regular people with active life style do not differ from that of an average adult individual. However certain amino acids and protein rich diets have been linked with reduced risk of obesity and metabolic diseases along with successful weight loss. These protein supplements have also been linked with maximization of anabolic reactions of skeletal muscles. However, the type and source of dietary protein supplements, timing of protein supplement intake and the intensity of exercise affects the effectiveness of the supplements to produce beneficial effects on muscle metabolism. There are some affirmation that intake of high amount of BCAA along with high fat diets may cause harmful effects on human metabolism. Combination of diets containing low amount of carbohydrates along with high amount of proteins and fats is found to show harmful effects on protein and amino acid metabolism and overall metabolic health. Thus the image which has been formed in the minds of common consumers about the role of protein supplements in getting fit and managing muscle growth and body mass is somewhat debatable and a good understanding of many physiological variables is required for the safe use and relevant utilization of proteins and amino acid supplements.

\section{Consumption Trends in Food \& Supplements Planned for Athletes}

Dietary supplements are made and targeted mainly at athletes to enhance their performance and intake of nutrition but maximum people who use these supplements are lifestyle users. European Union has defined sports person as "people who play or practice sports once a week or more" and lifestyle users as "people who play or practice sports less than once a week or not at all" but are consuming supplements made for athletes. Though these supplements are mainly focused on enhancing the performance of sports persons but the consequences of these supplements on the health of lifestyle users should also be kept in mind.

In developing countries the availability of cheap and counterfeit supplement products is a matter of great concern as they are not only less beneficial but are very harmful and hazardous. Another matter to be considered is the presence of prohibited substances in supplements such as those banned by the World Anti-Doping Agency (WADA) is found throughout the globe.

The need of protein increases with the time period and intensity of performance. An increased intake of energy to match the condition of higher physical activity will come with a higher consumption of protein. It is important to adapt the consumption of protein supplements according to the requirements of various training periods. Sufficient energy intake is another important issue, to make sure that the consumed amino acids are used up for the process of protein synthesis and are not oxidized. It important to keep in mind that the mold in fat consumption and dietary protein may have a higher effect than carbohydrates in optimizing body composition and body weight in sports persons, but this impact can differ according to the genetic variation in different individuals. The quality of dietary protein is also important. Protein with high biological value should be consumed.

Amino acids are not stored in human body like fatty acids and carbohydrates. This is a clear indication that one has to ensure daily consumption of amino acids needed for the synthesis of proteins and other metabolic functions is enough. Amino acids' level in the blood is relatively constant so if the dietary protein consumption is less than the required amount, there is an increase in the breakdown of muscle protein. Similarly in case of consumption of protein more than the required value, proteins are catabolized and used for energy. If there is lack of protein in the diet of an athlete, many effects may be observed on the organ system. Required amount of protein supports and helps in maintaining body 


\section{Food Science \& Nutrition Technology}

protein and bone metabolism, these parameters further enhance the performance of athlete and prevents injuries. In extreme cases, menstrual disorders are also reported in females due to lack of proteins. However, data regarding deficiency of protein in athletes is very less; there is very little information available on the adverse effects of prolonged intake of protein supplements.

\section{Conclusion}

There is diverseness among health authorities in their views on protein intake for athletes, sportspeople and sedentary people. There is a strong support by the scientific community on moderate protein consumption.

Diets rich in protein can help in weight loss but knowledge about the effects of prolonged consumption of proteins is still not sufficient. When a diet containing high amount of protein is required, in any case, special attention should be given to the overall food quality and origin of these proteins. The intake of ultra-processed foods is linked to the wide spread presence of many diseases. When it comes to amino acid and protein supplements the other substances present in them can cause harmful effects in case of prolonged intake of these supplements. We suggest that instead of adding protein or amino acid supplements in your diet, the protein in your diet should come from whole foods such as - fish, eggs, dairy products, legumes, cereals along with all the other necessary food components which maintain the overall well-being of the host. According to us protein and amino acid supplements should be used occasionally, only when the enhancement in athletic performance is difficult or not possible with a normal diet. Along with protein quality and quantity people should also maintain the proper balance of other components of food to ensure healthy life style and physical activities. 